\title{
Thilo Fehmel
}

\section{Konflikte erster und zweiter Ordnung in Europa}

In Europa geraten soziale Konflikte nach einer langen Phase ihrer national organisierten Einhegung wieder verstärkt in den Fokus. Insbesondere im Kontext der multiplen europäischen Krisen, die sich in ihrer Summe als Krise der europäischen Integration fassen lassen, werden in jüngster Zeit zunehmend soziale Konflikte thematisiert. Sie nehmen qualitativ neue Gestalt an, ${ }^{1}$ vor allem aber quantitativ und an Reichweite zu. Ursache dieser quantitativen Zunahme und der räumlichen Ausdehnung sozialer Konflikte sind die in Europa besonders intensiv stattfindenden Prozesse der Denationalisierung von Problemlagen. Folge der fortschreitenden Supranationalisierung politischen Handlungsdrucks und politischer Entscheidungen wie auch der fortschreitenden Transnationalisierung sozialer Beziehungen ist eine zunehmende Politisierung der europäischen Integration, ${ }^{2}$ also eine zunehmende Austragung von Konflikten im politischen Raum. Politisierung ist ein Indikator konflikthafter Strukturen; ${ }^{3}$ sie macht Konflikte sichtbar und deren Ursachen zum Gegenstand politischer Auseinandersetzung.

So einig sich sozialwissenschaftliche Kommentatoren mit Blick auf Europa über die Tatsache der Zunahme sozialer Konflikte sind, so uneinig sind sie sich in den Bewertungen dieser Entwicklung. Die Differenzen entzünden sich an den Einschätzungen der sozialen Folgen der Europäisierung sozialer Konflikte. Auf der einen Seite steht die Erwartung, dass die in der Krise der europäischen Vergemeinschaftung offensichtlich werdenden sozialen Konflikte das ohnehin »dünne Reservoir an gefühlten Gemeinsamkeiten in der und für die $\mathrm{EU}$ « noch weiter minimieren. Daraus wird die Empfehlung abgeleitet, die europäische Verflechtung »ein Stück weit zurück[zunehmen] «und den Subsidiaritätsgedanken zu stärken, ${ }^{4}$ letztlich also Abgrenzungsbemühungen mit Renationalisierungseffekten zu fördern. Dem steht die Ansicht gegenüber, dass gerade die Krisen Europas, die dadurch begünstigte Politisierung des Krisenmanagements und die darin zum Ausdruck kommende Transnationalisierung sozialer Konflikte in einen Integrationsschub hin zu europäischer Demokratisierung 5 und zu europäischer Vergesellschaftung ${ }^{6}$ münden.

1 Vgl. dazu Honneth 2012.

2 Lepsius 1999; Hooghe, Marks 2009.

3 Mouffe 2007, S. 35.

4 Vgl. Immerfall 2013, S. 203 ff.

5 Zürn 2013.

6 Vobruba 2013.

Leviathan, 42. Jg., 1/2014, S. 115 
Diese gegensätzlichen Auffassungen zu den Chancen europäischer transnationaler Vergesellschaftung - sozialer Konflikt statt soziale Integration vs. soziale Integration durch sozialen Konflikt - lassen sich in der generellen und keineswegs nur auf Europa bezogenen Frage zuspitzen: Wirken Konflikte sozialintegrativ oder sozialdesintegrativ? Begünstigen oder behindern sie die Bildung, Erhaltung und Weiterentwicklung von Gesellschaften? Zur Beantwortung dieser Fragen soll von sozialintegrativen Wirkungen in Anlehnung an Galtung ${ }^{7}$ dann die Rede sein, wenn Konflikte zu assoziativen Strukturen, also zu dauerhaft konstruktiven gegenseitigen Beziehungen - zu Simmels oft zitierten » positiven wechselseitigen Verhaltungsweisen $\ll^{8}$ - zwischen Akteuren führen oder diese, sofern sie bereits bestehen, nicht zerstören. Sozialdesintegrativ wären demgemäß Konflikte dann, wenn sich in ihrer Folge dissoziative Strukturen herausbildeten, also bestehende soziale Kontakte reduziert, wechselseitige soziale Beziehungen geschwächt und soziale Akteure voneinander isoliert würden. Derartige dissoziative Folgewirkungen sind nicht per se schlecht - es gibt Konstellationen, in denen die Separierung und Isolierung von Konfliktgegnern sinnvoll ist. Vergesellschaftung ist das jedoch nicht.

Angesichts der Komplexität moderner Gesellschaften sind einfache Antworten auf die gestellten Fragen nicht möglich. Ziel des vorliegenden Beitrags ist es vielmehr, grundlegende Argumente für Bedingungen zusammenzutragen, unter denen Konflikte sozialintegrative Wirkungen haben können (I. bis V.). Auf Basis der dabei gewonnen Erkenntnisse soll dann versucht werden, die gegenwärtig als verschärft wahrgenommenen Konfliktstrukturen in Europa zu bewerten und insbesondere daraufhin zu untersuchen, inwieweit diese wie auch die Krise Europas der europäischen Vergesellschaftung zu- oder abträglich sind (VI. und VII.).

\section{I.}

Moderne Gesellschaften sind Konfliktgesellschaften. Mehr noch: Folgt man Georg Simmel, dann sind Konflikte eine zentrale Form der Vergesellschaftung. Durch Auseinandersetzungen treten Akteure in soziale Beziehungen ein und interagieren miteinander. Durch die Wahrnehmung von Meinungs- und Interessenunterschieden bilden sich soziale Gruppen, die sich kommunikativ voneinander abgrenzen, indem sie sich aufeinander beziehen. Wenn sich die Meinungsunterschiede nicht darauf beschränken, differente soziale Positionen zu reflektieren, sondern darauf hinauslaufen, dass mindestens eine der beteiligten Seiten anstrebt, ihren relativen Status zu verändern, also ihren Zugang zu Macht oder zu knappen Ressourcen zu verbessern, dann wird aus simpler sozialer Ungleichheit ein einer Lösung zustrebender sozialer Konflikt. Konflikte sind aus dieser Perspektive Triebfedern sozialen Wandels. Sie stellen etablierte soziale Ordnungsgefüge infrage, nehmen deren reduzierte Komplexität zurück und lösen die damit verbundene Erwartungssicherheit auf.

7 Galtung 1970.

8 Simmel 1992 [1894], S. 58. 
Erst in der Moderne sind die Voraussetzungen dafür gegeben: Meinungsunterschiede gelten nicht als Ausdruck sozialer Pathologie; Auseinandersetzungen und offene Interessenverfolgung sind als Prinzip akzeptiert; Versuche von Akteuren, die jeweils eigenen Anschauungen als allgemeingültige Handlungsbegründungen durchzusetzen, sind als grundsätzlich legitim anerkannt, müssen sich aber andererseits auch Kritik, Infragestellung und die Konfrontation mit Gegenentwürfen gefallen lassen. Solche Auseinandersetzungen können heftig sein. Aber eine Gefahr für den Bestand einer Gesellschaft muss von derartigen modernen Praktiken nicht zwingend ausgehen.

\section{II.}

Anders als strukturfunktionalistische Theorien der sozialen Integration gehen soziologische Theorien des sozialen Wandels davon aus, dass Konflikte in modernen, pluralistischen Gesellschaften produktiv und konstruktiv sind beziehungsweise es unter bestimmbaren Bedingungen sein können. Um diese Bedingungen soll es im Weiteren gehen. Die integrative Wirkung sozialer Konflikte lässt sich mit vier Theoremen erklären.

Erstens: Mit dem Grad der funktionalen Differenzierung einer Gesellschaft steigt auch die Zahl von Zugehörigkeiten eines Individuums. Im Zuge dieser Pluralisierung von Zugehörigkeiten nimmt zugleich die Wahrscheinlichkeit von sozialen Konflikten zu. Dabei handelt es sich jedoch in der Regel um sich überschneidende Konflikte, die aus den Mehrfachzugehörigkeiten der Akteure resultieren. ${ }^{9}$ Mehrfachzugehörigkeiten relativieren die Bedeutung - und verringern so die Intensität sozialer Konflikte entlang einer einzigen, (vormals) zentralen Achse; ${ }^{10}$ sie verhindern Großpolarisierungen mit höherem Destabilisierungs- und Desintegrationspotenzial. Je größer und vielfältiger die Interdependenzen zwischen gesellschaftlichen Teilbereichen und sozialen Gruppen sind, desto eher sind relationale Positionen innerhalb eines einzelnen von vielen möglichen Interessengegensätzen Konfliktgegenstand ${ }^{11}$ und nicht grundsätzliche Fragen der Gesellschaftsorganisation.

Zweitens: Die Möglichkeit funktionaler Differenzierung moderner Gesellschaften wie auch die dadurch eröffnete Möglichkeit der Pluralisierung von Zugehörigkeiten ergeben sich aus dem Umstand, dass ein zunehmender Anteil von sozialen Konflikten innerhalb einer Gesellschaft teilbare Materien zum Gegenstand hat. ${ }^{12}$ Zumeist behandeln solche Konflikte Fragen der Verteilung des Sozialprodukts zwischen verschiedenen sozialen Gruppen; es sind also Verteilungskonflikte, Konflikte um das Mehr-oder-Weniger eines teilbaren und verteilbaren Gutes. Spiegelbildlich dazu sinkt mit fortschreitender gesellschaftlicher Modernisierung der Anteil kategorischer Konflikte des Entweder-Oder, deren Gegenstand unteilbare Materien und

9 Coser 1965.

10 Hirschman 1994.

11 Simmel 1992 b [1908], S. 846.

12 Hirschman 1994.

Leviathan, 42. Jg., 1/2014 
unvereinbare Werte sind, etwa entlang rivalisierender ethnischer, linguistischer oder religiöser Gruppenzugehörigkeiten. ${ }^{13}$ Diese Gruppenzugehörigkeiten werden auch in der Moderne nicht bedeutungslos, aber sie gehen auf in den erwähnten Mehrfachzugehörigkeiten, werden dadurch relativiert und mit den Verteilungslösungen von Verteilungskonflikten mehr oder weniger stark und mehr oder weniger dauerhaft deaktiviert. So gesehen, können die sich im Zuge gesellschaftlicher Differenzierung vollziehenden Säkularisierungsprozesse als grundlegende Transformation von Wertkonflikt- in Verteilungskonfliktstrukturen interpretiert werden. ${ }^{14}$ Verteilungskonflikte, also Konflikte um teilbare oder um verhandlungs-, ausgleichs- und gegebenenfalls substitutionsfähige Ressourcen oder politische Ziele, sind leichter beizulegen und daher deutlich weniger destabilisierend als Wertkonflikte. Verteilungskonflikte lassen sich per Kompromiss lösen. Kaum etwas ist so sozialintegrativ wie ein Kompromiss, für Simmel »eine der größten Erfindungen der Menschheit «. 15

Eine keineswegs triviale, aber oft übersehene unabdingbare Voraussetzung für die Lösung von Verteilungskonflikten ist die Existenz eines anerkannten und dem hohen Differenzierungsgrad moderner Gesellschaften angemessenen Verteilungsmediums. Als dafür besonders gut geeignet hat sich Geld erwiesen. Geld ermöglicht Modernisierung: Es ist »das Band, das die maximale Ausdehnung der [...] Gruppe mit der maximalen Differenzierung ihrer Mitglieder, nach der Seite der Freiheit und Selbstverantwortlichkeit, wie nach der qualitativ-arbeitsteiligen Differenzierung, in Beziehung setzt «. ${ }^{16}$ Mit Geld lassen sich soziale Konflikte leichter lösen. Das wiederum treibt funktionale Differenzierung und Interessenheterogenisierung voran und reduziert das Risiko gesellschaftsgefährdender Konflikte. Auch Macht ist teilbar, allerdings auf komplexere Weise. Das führt zu unserem dritten Punkt.

Drittens: Soziale Konflikte können integrativ wirken, wenn sie nach allseits anerkannten Regeln ausgetragen werden ${ }^{17}$ und wenn es im Falle von Verteilungskonflikten akzeptierte Verteilungsmechanismen gibt. Festlegungen zur Reichweite des Konflikts und seiner Lösung, Vorgaben zur Zulässigkeit von Methoden der Konfliktaustragung, kurz: die Verfahrensregeln der Konfliktaustragung sorgen dafür, dass Konflikte und ihre Lösungen bis zu einem gewissen Grad vorhersehbar und berechenbar sind, also nicht gesellschaftsgefährdend. Dazu gehört auch die Existenz der »Figur des durchsetzenden Dritten «18, einer Instanz, die die Austragung eines Konflikts überwacht und deren Intervention und Sanktion von den Konfliktbeteiligten akzeptiert wird. Dieses Setting aus Verfahrensregeln, Verteilungsmechanismen und Überwachungsinstanz nenne ich Konfliktrahmen. Innerhalb geltender,

\section{Aubert 1972.}

14 Taylor 2012.

15 Simmel 1992 a [1908], S. 375.

16 Simmel 1992 b [1908], S. 832.

17 Dahrendorf 1972, S. 41.

18 Knight 1997, S. 60. 
faktisch durchgesetzter und anerkannter Konfliktrahmen können Konflikte mit hoher Intensität ausgetragen werden, ohne die soziale Ordnung in ihrer Gesamtheit zu gefährden. Konfliktrahmen können vielfältige Formen annehmen. Allgemein aber gilt: Mit dem Übergang von vormodernen zu modernen, von überschaubaren zu komplexen Gesellschaften geht die Funktion der Konfliktrahmung von Moral und Sitte zu Recht und Gesetz über.

Viertens: Soweit ich sehe, ist man sich in den Sozialwissenschaften uneins über das Prinzip, das der Ausbreitung des Rechts als Konfliktrahmen zugrunde liegt. Zwar folgt die Ausdehnung des Rechts einem evolutionären Muster: Ohne dass es gesellschaftlichen Akteuren bewusst würde oder werden müsste, geht die fortschreitende Integration moderner Gesellschaften aus dem steten Erfahrungszuwachs im Umgang mit Konflikten hervor. ${ }^{19}$ Zugleich spielen aber starke Initiativimpulse eine wichtige Rolle für die Etablierung von Konfliktrahmen: Konfliktrahmen entstehen in Tragödien, in Krisenzeiten. Oder mit den Worten Alfred North Whiteheads: »Der erste Schritt zur soziologischen Weisheit besteht darin, anzuerkennen, dass die wichtigsten Fortschritte in der Zivilisation Prozesse sind, die die Gesellschaften, in denen sie stattfinden, beinahe zerstören «. ${ }^{20}$ Nur selten (beziehungsweise: immer seltener) sind damit Gesellschaften in toto gemeint. In funktional differenzierten Gesellschaften heißt das aber zumindest für gesellschaftliche Teilsysteme: Krisen begünstigen die Erschaffung und Verfeinerung von Konfliktrahmen. Unabdingbare Voraussetzung von Konfliktrahmen mit Mitteln des Rechts ist im Übrigen die Manifestation der zugrunde liegenden Konflikte, also deren Wahrnehmung oder Antizipation durch involvierte Akteure. ${ }^{21}$ Nur manifeste Konflikte sind der Konfliktregulierung, der Konfliktrahmung zugänglich.

In welchem Ausmaß soziale Konflikte sozial integrativ oder desintegrativ wirken, hängt also $\mathrm{ab}$

- vom Grad gesellschaftlicher funktionaler Differenzierung und von der Zahl der sich überlagernden - und neutralisierenden - Zugehörigkeiten eines Akteurs (strukturelle Dimension),

- von Konfliktmanifestoren (Krisen, Katastrophen oder anderen Impulsen und critical junctures) und vom manifesten Charakter des Konflikts (kognitive Dimension),

- davon, ob die am Konflikt Beteiligten den Gegenstand des Konflikts als teilbar oder nichtteilbar wahrnehmen und ob diesen Interpretationen entsprechend Kompromisslösungen des Konflikts möglich sind oder nicht (inhaltliche Dimension), und

- davon, ob für diese manifesten Konflikte anerkannte Konfliktrahmen existieren beziehungsweise ob die am Konflikt Beteiligten in der Lage sind, entsprechende Konfliktrahmen zu etablieren (institutionelle Dimension).

19 Dubiel 1997.

20 Whitehead 2000 [1927].

21 Dahrendorf 1958.

Leviathan, 42. Jg., 1/2014 


\section{Abbildung 1: Konfliktdimensionen}
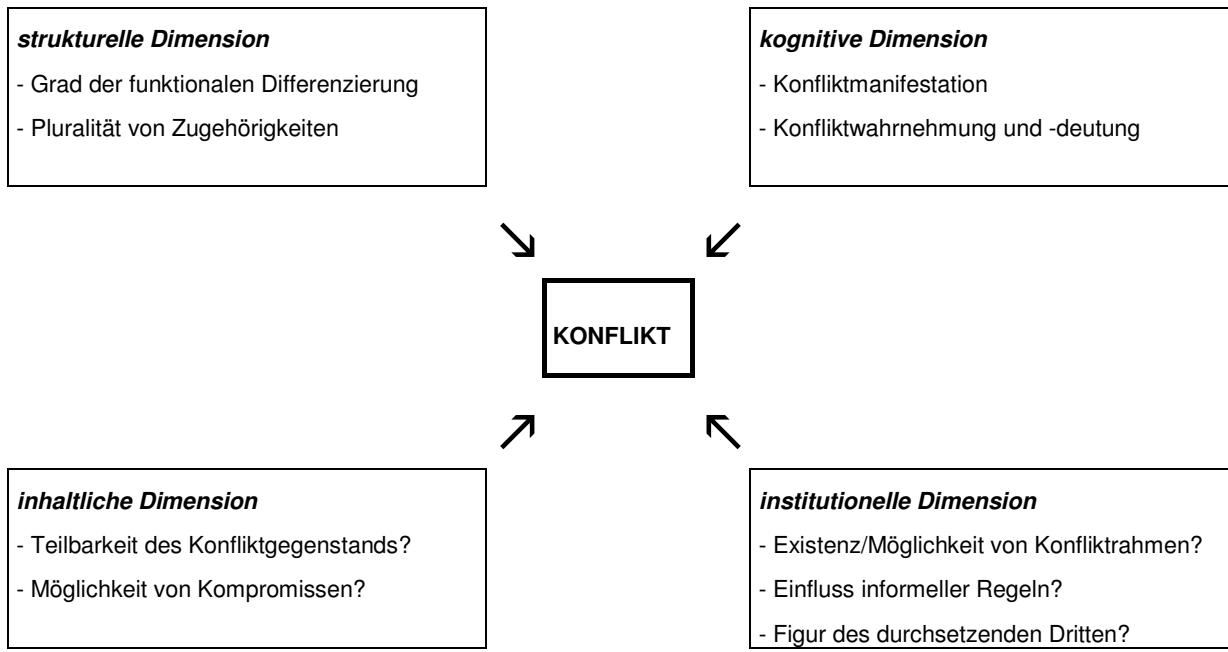

III.

Mit dieser Einteilung in vier Konfliktdimensionen, so denke ich, lässt sich grundsätzlich jeder soziale Konflikt analysieren Aber es bleiben offene Fragen. Ich konzentriere mich im Weiteren auf die Frage, wie Konfliktrahmen eigentlich genau entstehen - und wie sie sich wandeln.

Geht man davon aus, dass Konflikte Verteilungskonsequenzen haben, dann liegt es nahe, dass Akteure nicht nur innerhalb derartiger Konflikte nach Distributionsgewinnen streben. Sie streben auch danach, schon bei der Gestaltung eines relevanten Konfliktrahmens für derartige Konflikte Regelungen durchzusetzen, die dauerhaft Verteilungsvorteile in Aussicht stellen, indem sie die Möglichkeiten potenzieller Konfliktgegner einschränken - und zwar nicht nur situativ, sondern strukturell!22 Es leuchtet ja sofort ein, dass zum Beispiel Konflikte um die konstitutionellen Grundlagen einer politischen Gemeinschaft, also um einen basalen Konfliktrahmen, eine größere Tragweite haben als ein einzelner Konflikt, der im Rahmen dieser Verfassung ausgetragen wird. Daran zeigt sich: Auch Konfliktrahmen sind Gegenstand - und Ergebnis - von Konflikten. In solchen Konflikten geht es grundsätzlich darum, einerseits Regeln der zukünftigen Konfliktaustragung festzulegen und anderseits um die Frage, welchem Akteur eigentlich die Kompetenzen des »durchsetzenden Dritten « zugewiesen werden, also die Kompetenzen der Konfliktüberwachung und der Durchsetzung der Konfliktregeln.

Nimmt man die Möglichkeit von Konflikten um Konfliktrahmen ernst, dann ergeben sich Anschlussfragen. Die Festlegung eines Konfliktrahmens dient dem Eta- 
blieren eines typischen Musters des Umgangs mit einer Vielzahl gleichartiger einzelner Konflikte. Insofern hat sie deutlich weitreichendere Folgen als die Festlegung einer einzelnen Konfliktlösung. Es ist daher zu erwarten, dass die Einigung auf einen Konfliktrahmen um vieles schwieriger ist als die Lösung eines einzelnen konkreten Konflikts. Damit drängt sich erstens die Frage auf, ob Konfliktrahmen eigentlich teilbare oder nichtteilbare Konfliktgegenstände sind. Da von einem teilbaren Konfliktgegenstand dann die Rede sein soll, wenn für die Konfliktparteien ein anerkanntes und handhabbares Verteilungsmedium zur Kompromissfindung existiert, sind hier also Ressourcen gefragt, mit denen Konflikte um Konfliktrahmen beigelegt werden können. Nun ist zwar theoretisch nicht ausgeschlossen, dass es bei der Gestaltung von Regelsystemen zur Einhegung von Konflikten Kompromisse geben kann und dass Konfliktrahmen selbst eine verhandlungsfähige, substitutionsfähige Ressource sind. Allerdings ist gerade aufgrund ihrer Funktion, Konflikte wiederholt und dauerhaft zu strukturieren, die Wahrscheinlichkeit vergleichsweise groß, dass Konfliktrahmen eher den Charakter unteilbarer Materien haben. Das heißt aber auch, dass es sozialdesintegrative Wirkungen haben kann, wenn ihre Etablierung und Eingliederung in ein größeres, insgesamt ausgleichendes Institutionensystem nicht gelingt.

Darüber hinaus ist davon auszugehen, dass die Wahrnehmung von Knappheit oder Verfügbarkeit von Ressourcen nicht nur die Schärfe einzelner Konflikte, sondern auch die Gestaltung und Weiterentwicklung von Konfliktrahmen maßgeblich beeinflusst. Welche Bedeutung der Wahrnehmung von Ressourcen zukommt, verdeutlicht etwa ein kurzer vergleichender Blick auf Europa und die Vereinigten Staaten: Dass es im Jahrhundert der Industrialisierung nach ca. 1850 in den USA nicht zu vergleichbaren, jedenfalls nicht vergleichbar scharfen Klassen- und Verteilungskämpfen wie in Europa kam, lässt sich mit der in Amerika (nicht aber in Europa) bestehenden Möglichkeit des räumlichen Ausweichens bei Konflikten erklären. Das immer weitere Vordringen ins Landesinnere im 19. Jahrhundert zielte auch und nicht zuletzt auf die Erschließung neuer Ressourcen als Reaktion auf die sozialen Konflikte in den industriellen Zentren der nordamerikanischen Ostküste. In Europa hingegen zwangen die sozialen Konflikte im Zuge der Industrialisierung zu Konfliktregeln, die den differenten Verteilungsansprüchen und der fehlenden Möglichkeit räumlichen Ausweichens Rechnung tragen mussten. ${ }^{23}$

Für die Konfliktforschung ergibt sich aus diesem Beispiel zweitens die Frage, ob unter Bedingungen knapper werdender Ressourcen anders über Konfliktrahmen gestritten wird als unter Bedingungen leicht verteilbaren Wohlstands. Genauer gefragt: Liegt in knapper werdenden Ressourcen das Potenzial einer Demodernisierung dergestalt, dass aus Verteilungskonflikten Wertkonflikte werden und dass im Zuge dieser Entwicklung auch Konfliktrahmen zunehmend infrage gestellt werden? Plausibel ist das durchaus, und es liegt nahe, dass in einer Zeit, in der wohl die Wahrnehmung von Wohlstandsverlusten überwiegt, diese Frage an Bedeutung gewinnt. Mit zunehmender Knappheit einer Ressource steigt zunächst die Schärfe des Verteilungskonflikts, ab einem bestimmten Punkt dann aber auch die Wahr-

23 Offe 2005, S. 189 ff.

Leviathan, 42. Jg., 1/2014 
scheinlichkeit, dass bestimmte soziale Gruppen von der Verteilung dieser Ressource gänzlich ausgeschlossen werden. Die Erfahrung zeigt, dass die Gruppen, die über die Macht verfügen, andere von der Verteilung der knappen Ressource auszuschließen, diesen Ausschluss nicht mit Argumenten des relativen Bedarfs (Mehr-oderWeniger beziehungsweise Minderbedürftigkeit), sondern mit Argumenten der kategorialen Zugehörigkeit (Entweder-Oder beziehungsweise Minderwertigkeit) rechtfertigen. Zugehörigkeitsdeutungen und -entscheidungen orientieren sich an gemeinsam geteilten kulturellen Werten, zugleich werden diese zugehörigkeitsdefinierenden Werte zum handlungsleitenden Ausschlusskriterium. ${ }^{24}$ Der Ausschluss von der Verteilung eines Gutes wird also mit einem Wertargument begründet - und in diesem Sinne ist unter den Bedingungen knapper werdender Ressourcen und sehr ungleich verteilter Allokationschancen die Umwandlung von sozialintegrativen Verteilungskonflikten in unter Umständen sozialdesintegrative Wertkonflikte vorstellbar. Damit geraten dann zwangsläufig auch die Konfliktrahmen unter Druck, innerhalb derer vormals die Verteilungskonflikte ausgetragen wurden.

Wohin dieses Umschlagen von Verteilungskonflikten in Wertkonflikte und die entsprechende Anpassung eines Konfliktrahmens praktisch führen kann, schildert in frappierend realistischer Voraussicht im Sommer 1932 der zionistische Politiker Kurt Blumenfeld: Es waren vor allem »[...] der Weltkrieg, die übersteigerte Industrialisierung, die rasche Zunahme der Bevölkerung Europas, die Deklassierung der früher herrschenden und besitzenden Schichten, die ungeheuere Not der Massen, die Technisierung des Lebens [zusammen mit einem] grundlegenden Strukturwandel «, die dazu führten, dass ein Großteil der »Nachkriegsgeneration immer weniger imstande war, ihr eigenes Leben zu gestalten und sich mit irgendeiner Wahrscheinlichkeit auf einen ihm einen gesicherten Platz bietenden Beruf vorbereiten zu können «. Diese präzise beschriebenen Verteilungskonflikte werden in Deutschland aber als Wertkonflikte, als hochgradig dissoziativer »deutsch-jüdischer Kulturkonflikt « ausgetragen, bei dem »Angehörige nationaler Minderheiten [...] Bürger minderen Rechts sind « und »jede Aeußerung über Juden [...] eine Verunglimpfung und Minderwertigkeitserklärung des Judentums [ist]. Der Mord an dem Menschen anderer Rasse und besonders am Juden wird verherrlicht «. ${ }^{25}$

Die rechtliche »Zurückstufung «, die im Nationalsozialismus an den für diesen Zweck »neudefinierten " Juden vollzogen wurde und die in beispielloser Weise zuerst der Enteignung, dann der Ghettoisierung, schließlich der Deportation und Vernichtung den Weg ebnete, bedurfte der Stilisierung und Instrumentalisierung eines Wertkonflikts. ${ }^{26}$ Ausmaß und Gründlichkeit, mit denen im Nationalsozialismus einer Kategorie von etablierten Staatsbürgern die Bürgerrechte entzogen wurden, sind einmalig. Doch auch in der Gegenwart lassen sich in der Form von Wertkonflikten strukturierte Konstellationen finden. Das führt drittens zur Frage: Gibt es eigentlich eine Figur des durchsetzenden Dritten für Konflikte um Konfliktrah-

\section{Walzer 1992.}

25 Blumenfeld 1932, S. 353 f.

26 Steinert 2007. 
men? Über welche Befugnisse müsste dieser verfügen, damit Konflikte um Konfliktrahmen geordnet ablaufen und - nicht zuletzt - konsensual einen durchsetzenden Dritten für den fraglichen Konfliktrahmen selbst festlegen? Diese Fragen deuten einen infiniten Regress an. Ich vermute, dass es diese Feststellung war, die Whitehead und andere zur Betonung der initiierenden Katastrophe veranlasst hat. Zweifellos begünstigen schwerwiegende politische und gesellschaftliche Krisen den Einfluss externer Akteure. Diese können dann, im Stile des durchsetzenden Dritten und mit entsprechender Macht ausgestattet, Institutionensysteme radikal umbauen, also auch Konfliktrahmen etablieren oder vollkommen neu ausrichten, ohne dabei allzu viel Rücksicht auf frühere institutionelle Strukturen und Erfahrungshaushalte nehmen zu müssen. Die unterschiedlichen Nachkriegs-Entwicklungen in West- und Ostdeutschland infolge ihrer Aufteilung auf getrennte Machtsphären externer Akteure zeigen dies beispielhaft. Und in historischen Abhandlungen zum Entstehungskontext des deutschen Grundgesetzes, eines zentralen Konfliktrahmens, wird nie der Hinweis vergessen, unter welch prägendem Eindruck die Mitglieder der verfassungsgebenden Versammlung angesichts der Erfahrungen mit der kurz zuvor überwundenen Diktatur standen.

IV.

Keineswegs immer aber geht der Etablierung eines Konfliktrahmens eine derart tiefgreifende Katastrophe voraus. Verbreiteter dürfte der Fall sein, dass die Schaffung formaler Konfliktrahmen - zumindest partiell - ein Prozess der Kodifizierung informeller Regeln ist. In einer statischen Perspektive lässt sich sagen: Formale Konfliktrahmen basieren auf ihnen zugrunde liegenden informellen Werten, Überzeugungen, Regeln. ${ }^{27}$ Dynamisch formuliert: Die Chance, dass de jure existierende, kodifizierte Konfliktregeln auch faktisch durchgesetzt sind und dass sie das (Konflikt-)Verhalten gesellschaftlicher Akteure strukturieren, steigt in dem Maße, in dem sie mit bestehenden informellen, tradierten, internalisierten gesellschaftlichen Regeln korrespondieren. ${ }^{28}$

Die Pluralisierung von Zugehörigkeiten individueller oder kollektiver Akteure wirkt zwar gesellschaftsbildend, weil in ihrem Zuge die Zahl sozialer Konflikte zu-, die Bedeutung des einzelnen Konflikts jedoch abnimmt. Das setzt allerdings voraus, dass sich aus dieser Zugehörigkeitspluralisierung manifeste, also den Akteuren bewusste Konflikte ergeben. Denn nur dann ergibt sich auch ein politischer Druck der Konfliktregulierung und der Etablierung von Konfliktrahmen. Dies führt zu der Einsicht, dass längst nicht jede weitere Ausdifferenzierung und Pluralisierung von Zugehörigkeiten zu neuen, gar manifesten Konflikten führen muss. Das gilt insbesondere dann, wenn bereits Konfliktregeln und Verteilungsmechanismen existieren, mit denen prinzipiell auch neue oder veränderte Konfliktkonstellationen bearbeitet werden können. Dass sich im Zuge gesellschaftlicher Modernisierung individuelle

27 Knight 1997, S. 93.

28 Voigt 2013.

Leviathan, 42. Jg., 1/2014 
Rollen vervielfältigen, erzwingt also nicht unbedingt neue Konfliktrahmen, denn die Rahmen für die möglichen Konflikte, in die das Individuum aufgrund seiner - zahlenmäßig zunehmenden - Zugehörigkeiten gerät oder geraten kann, existieren ja oft bereits.

Daraus folgt (gleichsam als Entgegnung auf und in Ergänzung von Simmel): Nicht neue Konflikte per se, sondern die Wahrnehmungen bestehender Konfliktrabmen als unangemessen und ungeeignet für die Regulierung der neuen Konflikte erhöhen den Druck zur Anpassung existierender oder zur Schaffung neuer Konfliktrahmen. Auslöser dieser Wahrnehmung kann einerseits exogenes Geschehen sein, das unerwartet die Konfliktbedingungen massiv modifiziert und damit einen etablierten Konfliktrahmen infrage stellt. Handlungstheoretisch interessanter ist jedoch der endogene Fall, dass Konfliktrahmung selbst soziale Konflikte erzeugt oder restrukturiert, was weitere Konfliktrahmung erforderlich macht - Regulierungswirkungen werden Anlass zu neuen Regulierungsmotiven. Der zunehmende Bedarf an Konfliktregulierung ist also Folge vorheriger, über kurz oder lang als unzureichend erkannter Konfliktregulierung. Georg Vobruba nennt diesen Mechanismus defizitäre Institutionalisierung. ${ }^{29}$ Dieser Mechanismus ist jedoch weder teleologisch, noch folgt er einer funktionalistischen Gesetzmäßigkeit. Denn ob und wie der Bedarf an endogen veranlasster Konfliktregulierung befriedigt wird, ist eine empirische Frage. $\mathrm{Zu}$ deren Beantwortung sind subjektiv interpretierte situative Interessenkonstellationen und Machtverhältnisse in den Blick zu nehmen: Institutionenwandel ist die Folge der Verschiebung von Verhandlungsmacht. ${ }^{30}$ Die Revision eines Konfliktrahmens ist eine Reaktion auf seine wahrgenommene Ineffizienz. Das Ergebnis der Revision ist eine Restrukturierung der Konfliktbeziehung, nicht aber zwangsläufig auch eine aus Sicht aller Beteiligten effizientere Konfliktregulierung. ${ }^{31}$

\section{V.}

Unabhängig davon, wie man die gestellten Fragen beantwortet und die angesprochenen Punkte weiterverfolgt: In ihrer Gesamtheit machen sie aufmerksam auf einige kategoriale Unterschiede zwischen einfachen Konflikten einerseits und Konflikten um Konfliktrahmen andererseits. Es ist daher sinnvoll, zwischen Konflikten erster und zweiter Ordnung zu unterscheiden. Aus dem Umstand, dass - wie beschrieben - auch Konfliktrahmen Gegenstand und Ergebnis von Konflikten sind, ergibt sich ein dynamisches Modell eines Konfliktzyklus, das sich in die folgenden Phasen unterteilen lässt:

Wir haben es in Phase 1 zu tun mit der Manifestation eines Konflikts, also mit der Entstehung eines Konflikts erster Ordnung. Im Zuge der Konfliktlösung kommt es in Phase 2 zur Wahrnehmung des Bedarfs an dauerhafter Einhegung des Konflikts, also an der Etablierung eines Konfliktrabmens. Beim Versuch der Einhegung zeigen

29 Vobruba 2012.

30 Knight 1997, S. 188.

31 Hooghe, Marks 2009, S. 2. 
sich in Phase 3 unterschiedliche Positionen, es manifestiert sich also ein Konflikt um den Konfliktrahmen beziehungsweise ein Konflikt zweiter Ordnung. Phase 4 umfasst die Lösung dieses Konflikts zweiter Ordnung und die Etablierung eines Konfliktrahmens, dies ist also die Phase der Institutionalisierung. Im Laufe der Zeit verändern sich in Phase 5 die Verteilungseffekte des Konfliktrahmens, vielleicht verändert sich auch nur die Bewertung der Verteilungseffekte durch einzelne Akteure. Ursache dieser Veränderungen können die Verknappung teilbarer Ressourcen sein, die Veränderung von Interessen, die Verschiebung von Machtverhältnissen oder auch der Wandel von Zugehörigkeiten. Als Folge dieser Veränderungen kommt es in Phase 6 zur Entwicklung neuer Konflikte oder zur Re-Manifestation eines bislang befriedeten Konflikts, also zu einem Konflikt erster Ordnung. Im Zuge dieser Entwicklung erweist sich in Phase 7 der bestehende Konfliktrahmen als defizitär; betroffene Akteure nehmen ihn als immer weniger angemessen wahr, sie stellen ihn zunehmend in Frage; das führt zu Konflikten um den Konfliktrahmen, also zu Konflikten zweiter Ordnung. Sofern es gelingt, diesen Konflikt um den Konfliktrahmen zu lösen, kommt es in Phase 8 zur Anpassung des Konfliktrahmens (also zu ergänzender Institutionalisierung) - und der gleichsam spiralförmige Zyklus beginnt über kurz oder lang mit Phase 5 von vorn.

Insgesamt zeigt sich damit: Konflikte erster und zweiter Ordnung gehen der Institutionenbildung voraus - und früher oder später auch aus ihr hervor! Um auf Simmel und auf meine grundlegende Eingangsfrage zurückzukommen: Konflikte wirken dann vergesellschaftend, wenn sie zur Herausbildung, Institutionalisierung und Weiterentwicklung von Konfliktrabmen führen.

\section{VI.}

Mit diesen generellen Überlegungen zur Differenzierung grundsätzlicher Konfliktdimensionen und zur Entwicklung des Modells eines Konfliktzyklus wende ich mich nun dem Problem europäischer Vergesellschaftung zu. Sind wir auf dem Weg zu einer europäischen Gesellschaft? Welche Bedeutung haben soziale Konflikte in diesem Zusammenhang? Und welche Rolle spielt dabei die Krise Europas?

Das Projekt der europäischen Integration war über Jahrzehnte hinweg nahezu ausschließlich ein politisches Projekt der Institutionenbildung zum Zwecke der Schaffung eines gemeinsamen Marktes. Mit fortschreitender politischer und/oder ökonomischer Integration Europas entwickeln und verstärken sich vor allem seit dem Vertrag von Maastricht zwangsläufig auch interdependente grenzüberschreitende Beziehungen zwischen sozialen Akteuren. Exakt das war und ist die übergreifende Intention des Projekts Europa und all seiner einzelnen Integrationsprojekte. Die Schaffung des Schengen-Raums oder der Eurozone, die Initiierung des Bologna-Prozesses, die Durchsetzung der Unionsbürgerschaft, der Arbeitnehmerfreizügigkeit oder der Niederlassungsfreiheit sind prägnante Beispiele. Ihnen ist das Potenzial gemeinsam, die nationale Schließung von Teilsystemen und Protektionsräumen (Arbeitsmärkten, Sozialsystemen, Währungsräumen, Gütermärkten, politischen Institutionensystemen etc.) aufzuheben oder zu relativieren. Sie erhöhen auf 
je spezifische Weise die Chancen auf grenzüberschreitende soziale Beziehungen, Relationierungen und Interdependenzen. ${ }^{32}$ Sie errichten so potenzielle europäische Sozialräume. Eine Vielzahl von Bewegungen und Handlungen sozialer Akteure in diesen Sozialräumen - als grenzüberschreitend mobile Arbeitskräfte, Konsumenten oder Studierende, als europaweit agierende Unternehmen, als europaweit asylsuchende Drittstaatsangehörige, als Wähler eines gemeinsamen europäischen Parlaments oder als Nutzer einer gemeinsamen Währung - hat grenzüberschreitende Handlungsfolgen. Damit werden zugleich Unvereinbarkeiten von Interessen, Zielen, Mitteln, Verhaltensweisen oder Einstellungen sozialer Akteure wahrscheinlicher, die in ihrer Ausdehnung beziehungsweise Reichweite die etablierten nationalen Konfliktrahmen überschreiten. Das heißt: Aus potenziellen wechselseitigen transnationalen sozialen Beziehungen werden faktische in dem Maße, wie sie manifeste soziale Konflikte - und damit: neue Regelungsbedürfnisse - hervorrufen. Mehr noch als die bloßen Verdichtungen sozialer Beziehungen können die damit einhergehenden Konflikte zu europäischen Integrationsschüben werden.

Das Konfliktpotenzial ist nicht in allen europäischen Sozialräumen gleich stark ausgeprägt. Es ist in jenen Sozialräumen relativ gering, in denen entweder nur vergleichsweise wenige Akteure agieren (Beispiel: europäischer Hochschulraum) oder in denen das Handeln der Akteure von nur minderer Bedeutung für die Lebenswelt dieser Akteure ist (Beispiel: europäischer Raum der politischen Partizipation). Groß ist das Konfliktpotenzial hingegen, wenn viele Akteure betroffen sind und wenn es um viel geht (zum Beispiel in der Eurozone oder in den Protektionsräumen für Produktmärkte, Arbeitsmärkte und Sozialsysteme). Diese »neuen «, grenzüberschreitenden Konflikte sind - in der oben eingeführten Terminologie - Konflikte erster Ordnung. Sie entstehen als Folge vorheriger Institutionenbildung auf europäischer Ebene (Schengen-Raum, Eurozone, Europäischer Binnenmarkt etc.), die sich im Laufe der Zeit beziehungsweise unter bestimmbaren Bedingungen als unzureichend für die transnationale Verteilung der Institutionalisierungsfolgen erwiesen hat. Aus diesen Wahrnehmungen entwickeln sich unterschiedliche Bedürfnisse nach ergänzender Institutionalisierung, und im Rahmen von Konflikten zweiter Ordnung wird verhandelt, inwieweit diesen unterschiedlichen Bedürfnissen supranational regulativ entsprochen wird. ${ }^{33}$

Die Einwände gegen diese Einschätzung der Möglichkeiten supranationaler ergänzender Institutionalisierung sind ebenso bekannt wie gewichtig: Es gibt kein den nationalen Systemen vergleichbares europäisches Parteiensystem, kein europäisches Wahlsystem und keine politische Öffentlichkeit europäischer Reichweite. Es gibt kein europäisches System zur Strukturierung der Arbeitsbeziehungen, kein gemeinsames Staatsverständnis und keine Einigkeit über Politikstile. Stattdessen gibt es all dies in je national unterschiedlichen Varianten. ${ }^{34}$ Diese Befunde sind für die europäische Vergesellschaftung in der Tat ein neuralgischer Punkt. Denn es ist

32 Wobbe 2009.

33 Zürn 2013.

34 Vgl. für viele Bach 2009. 
keineswegs ausgemacht, dass die Manifestation und Intensivierung grenzüberschreitender sozialer Konflikte automatisch auch zu grenzüberschreitend organisierten Konfliktlösungsstrategien und gar zu grenzüberschreitend geltenden Konfliktaustragungsregularien führt. Europäische Integration durch europäisches Recht ${ }^{35}$ ist nur eine Möglichkeit. Eine andere besteht im Versuch, die sozialen Folgen der zunehmenden Konflikte als Nebenprodukt der europäischen Integration - fast möchte man sagen: wie gewohnt - innerhalb nationaler Rahmen zu bearbeiten. In diesem Falle adressieren soziale Akteure ihre Konfliktregelungserwartungen gerade nicht an die europäische Ebene, stattdessen richten sie ihre Forderungen nach Konfliktentschädigung und Integrationsfolgenkompensation an die jeweils nationale Ebene. ${ }^{36}$ Grenzüberschreitende soziale Konflikte werden also entschärft, indem sie in nationale Sozial- und Umverteilungssysteme umgeleitet werden. Leistungsfähige nationale Sozial- und Umverteilungssysteme sind aus dieser Sicht Interdependenzunterbrecher; sie trennen die sozialen Relationen, die sich im Zuge der politischen Integration Europas bilden. Europäische Vergesellschaftung ergibt sich daraus nicht. (Inwieweit dieses Trennverfahren zur Zufriedenheit aller Erwartungsträger gelingt, ist freilich eine empirische Frage; im Kontext der gegenwärtigen Krisen wird auf diese Frage der Leistungs- und Kompensationsfähigkeit der nationalen Umverteilungssysteme zurückzukommen sein.)

Dessen ungeachtet ist die Chance vergleichsweise groß, dass jeder weitere Schritt innerhalb des beschriebenen Wechselspiels aus trans- beziehungsweise supranationalen Konflikten erster und zweiter Ordnung die wechselseitigen grenzüberschreitenden Beziehungen zwischen Akteuren assoziativ verdichtet. Das ist deshalb so, weil im Zusammenspiel der oben eingeführten vier Konfliktdimensionen im europäischen Kontext grundsätzlich recht günstige Bedingungen vorliegen. Mit Blick auf die strukturelle Dimension der Gesellschaftsbildung durch Konflikt ist ein Zusammenhang von Belang, auf den mit bemerkenswerter Voraussicht bereits Georg Simmel aufmerksam gemacht hat: Die aus funktionaler Differenzierung erwachsende Heterogenisierung innerhalb von Gruppen oder Gesellschaften führt zu zunehmender Homogenität zwischen solchen Gruppen oder Gesellschaften. ${ }^{37}$ Dadurch verwischen Gruppengrenzen, es »entstehen neue soziale Verbände, die vielfach über die Gruppengrenzen hinausreichen «, und es vollzieht sich ein »Prozeß der Skalenvergrößerung « ${ }^{38}$ Europa ist dafür das Paradebeispiel: Im Zuge der europäischen Integration vervielfältigen sich nicht nur, wie geschildert, individuelle Zugehörigkeiten zu transnationalen Sozialräumen, sondern es differenzieren sich generell die europäischen nationalen Gesellschaften im Zuge ihrer Modernisierung immer weiter aus - und werden sich damit strukturell immer ähnlicher. ${ }^{39}$ Die Konsequenz:

\section{Münch 2008 a.}

36 Jessop 2010.

37 Simmel 1992 b [1908].

38 van der Loo, van Reijen 1992, S. 116.

39 Münch 2008 b; Fehmel 2013.

Leviathan, 42. Jg., 1/2014 
»[D]ie Differenzierung und Individualisierung lockert das Band mit den Nächsten, um dafür ein neues - reales und ideales - zu den Entfernteren zu spinnen «. ${ }^{40}$

Dass das vollkommen konfliktfrei geschieht, ist unwahrscheinlich. Aber gemäß der oben eingeführten inhaltlichen Dimension der Gesellschaftsbildung durch Konflikt muss das der Bildung einer europäischen Gesellschaft keineswegs abträglich sein. Mit der Pluralisierung von Zugehörigkeiten in und durch Europa nehmen soziale Konflikte zu, zugleich aber überlagern und überschneiden sie sich zunehmend und verlieren allein schon dadurch an grundlegendem Dissoziierungspotenzial. Hinzu kommt, dass es sich bei den erwähnten transnationalen sozialen Konflikten ihrem Wesen und ihren Gegenständen nach primär um Verteilungskonflikte handelt. Im grenzüberschreitenden Streit um Arbeits-, Absatz-, Konsum- oder Studienmöglichkeiten und -bedingungen geht es nicht um miteinander unvereinbare Werte, sondern um das Mehr-oder-Weniger (ver)teilbarer - und damit: um für Kompromisse zugängliche - Güter. Nochmals begünstigt wird diese Kompromissfähigkeit der Mehrzahl grenzüberschreitender Konflikte durch den Euro als gemeinsame, sozialraumstrukturierende Währung, also durch die Existenz eines Verteilungsmediums, das den Wechselkursmechanismus als Interdependenzunterbrecher abgelöst hat und soziale Akteure in zuvor nicht gekannter Weise zwingt, sich grenzüberschreitend miteinander auseinanderzusetzen. ${ }^{41}$

Noch am ehesten wertebasiert ist das grenzüberschreitend interdependente politische Handeln der Unionsbürger als Wähler eines gemeinsamen europäischen Parlaments wie auch als Wähler nationaler Regierungen. ${ }^{42}$ Aber auch hier überwiegen die Chancen der Sozialintegration die Gefahren der Dissoziierung. Kompromisslösungen zwischen politischen Orientierungen sind nicht zuletzt aufgrund der Einübung des Umgangs mit dieser Konfliktlinie in nationalen Rahmen die Regel. Divergente kulturelle Orientierungen äußern sich darüber hinaus in bestimmten, soziostrukturell erklärbaren Einstellungsverteilungen gegenüber Europäern anderer Nationalität. Inwieweit ablehnende bis xenophobe Einstellungen Ausdruck kulturell überformter Verteilungskonflikte oder Ausdruck originärer, verteilungsunabhängiger Wertkonflikte sind, lässt sich nur selten klar bestimmen. Unbestritten aber gewinnt die Frage sozialer Diskriminierung angesichts zunehmender europäischer Binnenwanderung für die Erörterung der Chancen europäischer Vergesellschaftung an Relevanz - und ihr wird supranational mit einem institutionalisierten und regelmäßig zumindest nominal durchgesetzten Gleichheitsgrundsatz begegnet.

Damit rückt die institutionelle Dimension der Gesellschaftsbildung durch Konflikt ins Blickfeld. In dem Maße, wie diverse Schließungsmechanismen nationaler Teilsysteme und Protektionsräume aufgebrochen und ihrer Funktion als Interdependenzunterbrecher enthoben werden, in dem Maße steigt der Bedarf an transnationaler Konfliktrahmung. In und für die Europäische Union existiert ein ausge-

40 Simmel 1992 b [1908], S. 795.

41 Vobruba 2012; Schroeder, Weinert 2003.

42 So sind etwa die Ergebnisse der jüngsten Bundestagswahl, der eigenen Betroffenheitswahrnehmung folgend, in einigen europäischen Ländern mit mindestens ebenso großem Interesse beobachtet worden wie in Deutschland selbst. 
bautes Institutionensystem, dem diese Funktion der Regulierung und Rahmung transnationaler Konflikte zukommt, und es gibt mit der Europäischen Kommission und mehr noch mit dem Europäischem Gerichtshof Akteure, die die Rolle der Figur des durchsetzenden Dritten innehaben. Stärker als in national geformten Konfliktkulturen ist der zentrale Durchsetzungsmechanismus auf europäischer Ebene das Recht. Es basiert auf den europäischen Verträgen und ist damit »eine rationale, von Traditionen unabhängige, allein auf die Funktion der Gewährleistung des geordneten Zusammenlebens freier Individuen bezogene Konstruktion « ${ }^{43}$

Das ist oft problematisch, und Kollisionen zwischen europäischer Rechtsetzung und nationalen Rechtstraditionen sind nicht selten: Anders als üblicherweise nationale Rechtskulturen ist formales europäisches Recht eben nicht das Resultat der Kodifizierung informeller Regeln. Man kann, soweit die Rechtsetzung eingespielte nationale Verteilungsmuster betrifft, daraus die Forderung ableiten, nationale Protektionsräume zu schützen, um transnationale Verteilungskonflikte zu vermeiden, ${ }^{44}$ muss sich aber darüber im Klaren sein, dass diese Schließungsneigung renationalisierende, mithin dissoziative Konsequenzen hat, die der europäischen Idee diametral entgegenstehen. Hält man hingegen an der Grundintention des Projekts Europa, der Vertiefung interdependent grenzüberschreitender Beziehungen zwischen sozialen Akteuren, fest, dann sind kodifizierte Konfliktregeln erforderlich, die gerade das transnationale (Konflikt-)Verhalten gesellschaftlicher Akteure strukturieren. Solange aber auf europäischer Ebene der Zustand rudimentärer Parlamentarisierung und Politisierung ${ }^{45}$ anhält, kommt die Erarbeitung dieser Konfliktregeln primär dem Europäischen Gerichtshof zu. Und gerade weil - beziehungsweise: solange - es kein den nationalen Systemen vergleichbares europäisches politisches System der Konfliktregulierung gibt, ist die oft beklagte politische Macht des Europäischen Gerichtshofs so groß: Es fehlt die glaubwürdige Drohung, dass seine Entscheidungen durch Politik revidiert werden könnten. ${ }^{46}$ Soll dem abgeholfen werden, wäre also nicht nationale Schließung, sondern transnationale Politisierung zu fordern. Ich komme darauf zurück.

Die Überlegungen zu den strukturellen, inhaltlichen und institutionellen Voraussetzungen einer europäischen Gesellschaftsbildung durch Konflikte zusammenfassend, zeigt sich: Als unvermeidbare Nebenfolge der europäischen Integrationsprojekte nehmen transnationale soziale Konflikte zu, sie haben ganz überwiegend den Charakter von Verteilungskonflikten, und es bestehen günstige institutionelle Bedingungen für das Wechselspiel aus Konflikten erster und zweiter Ordnung. In dem Maße, wie die politisch-ökonomische Integration Europas zu sozialen Konflikten führt, die einen über den Nationalstaat hinausgehenden Konfliktrahmen erforderlich machen, in dem Maße vollzieht sich europäische Vergesellschaftung. Europa wird zum Konfliktraum.

43 Münch 2008 b, S. 21.

44 Vgl. Lamping 2010; Immerfall 2013.

45 Bach 2009.

46 Höreth 2013.

Leviathan, 42. Jg., 1/2014 


\section{VII.}

Eine Konfliktdimension blieb im Kontext europäischer Vergesellschaftung bislang undiskutiert. Voraussetzung der sozialintegrativen Konfliktrahmung und -regulierung ist die Manifestation der zugrunde liegenden Konflikte. Konflikte sind manifest, wenn sie von involvierten Akteuren selbst als solche wahrgenommen oder antizipiert werden, wenn die Konfliktinvolvierten im Wesentlichen bekannt sind und wenn mindestens ein Konfliktbeteiligter seinen Bedarf an einer Konfliktlösung artikuliert und gegebenenfalls eine entsprechende Forderung der Konfliktregulierung an einen durchsetzenden Dritten adressiert. Das ist Gegenstand der kognitiven Dimension der Gesellschaftsbildung durch Konflikt.

Besonders deutlich wahrnehmbar sind Konfliktkonstellationen in bedrohlichen sozialen Situationen, in denen bei gegebenen situativen Anforderungen die zur Verfügung stehenden Bewältigungsstrategien als unzureichend empfunden werden und sich unter dem Eindruck zu knapper Ressourcen subjektive Erfahrungen des individuellen und kollektiven Kontrollmangels ausbreiten. Die multiplen Krisen der europäischen Integration entsprechen diesem Muster. Eine Krise ist mit Friedrichs die wahrgenommene Gefährdung eines institutionalisierten Handlungsmusters. ${ }^{47}$ Institutionelle Ineffizienzen sind, wie gezeigt, nicht ungewöhnlich, sie rufen im Normalfall inkrementelle Anpassungsversuche hervor. Zu Krisen werden institutionelle Ineffizienzen, wenn sie unter den Bedingungen besonders abrupter und/oder besonders massiver Veränderungen (der Wahrnehmung) von Verteilungseffekten eines Konfliktrahmens zu Reaktionen und Gestaltungsversuchen führen, die von relevanten betroffenen Akteuren aus dem einen oder anderen Grund als inkonsistent wahrgenommen werden. Grundsätzlich gilt: Inkonsistenzen stoßen Deutungswandel an, ermöglichen über neue Interpretationsmuster institutionelle Innovation und schaffen auf diese Weise neue Gelegenheitsstrukturen zur Verfolgung von Interessen. In diesem Sinn sind Krisen Aktualisierungen von Gesellschaftsbezügen. Ebenso grundsätzlich gilt aber auch: In solchen Konstellationen herrscht ein Überschuss an Situationsdiagnosen und interessengeleiteten, weil selbstbezüglichen Handlungsempfehlungen; zugleich besteht gesteigerter Rechtfertigungsbedarf für jede dieser Krisendiagnosen und jede dieser Handlungsempfehlungen. Krisen zeichnen sich also aus durch institutionell unterbestimmte Handlungsbedingungen einerseits, durch konkurrierende Interpretationen bezüglich der angemessenen institutionellen Regulierung, zuweilen auch bezüglich der Legitimität der sie tragenden Akteure andererseits. ${ }^{48}$ In den Begrifflichkeiten der vorangegangenen Darlegungen sind Krisen damit Phasen, in denen Konfliktrahmen besonders nachdrücklich zur Disposition stehen. Aus konflikttheoretischer Perspektive ergeben sich Krisen aus der Häufung und Intensivierung von Konflikten zweiter Ordnung.

Für die Europäische Union scheinen solcherart definierte Krisen der bevorzugte Integrationsmodus zu sein. ${ }^{49}$ Und genau das lässt sich in Europa auch zurzeit beob-

47 Friedrichs 2007, S. 14.

48 Vobruba 2013.

49 Kühnhardt 2009. 
achten. Die gegenwärtige komplexe Konstellation der europäischen Staatsschuldenund Währungskrise nahm ihren Anfang nicht etwa in den Schockwellen, die die Immobilienkrise in den USA aussandte, sondern in der defizitären Institutionalisierung der europäischen Wirtschafts- und Währungsunion selbst. In ihren institutionellen und sozialen Folgen geht die Eurokrise aber inzwischen über Fragen der Währungs- und Fiskalpolitik weit hinaus und macht konfliktuöse soziale Relationen manifest, die in der Wirtschafts- und Währungsunion (WWU) von Beginn an strukturell angelegt sind. Darum soll es in den abschließenden Überlegungen gehen.

Die Geschichte der Schulden- und Währungskrise lässt sich zerlegen in viele einzelne Sequenzen von Konflikten um Konfliktrahmen, bei denen unterschiedliche grundlegende Ideen und Ideologien miteinander um politische Dominanz konkurrieren - und darum, die Verteilungswirkungen konkreter Konflikte dauerhaft zu strukturieren. ${ }^{50}$ Verhandelte Fragen waren beziehungsweise sind etwa: Welches Ausmaß soll die politische Unabhängigkeit der Europäischen Zentralbank (EZB) haben? Sollen sich die Staaten der Eurozone gemeinsam (mit Eurobonds) oder je separat (zu dann sehr unterschiedlichen Bedingungen) am Kapitalmarkt refinanzieren? Erfordert die einheitliche Währung eine einheitliche Fiskalpolitik oder nicht? Und wer ist eigentlich befugt zur Bewertung der Zahlungsfähigkeit von Staaten?

Nicht alle diese und andere Fragen sind neu, aber sie überschreiten in ihrer verschärften Umstrittenheit im Zuge der Finanzkrise die Wahrnehmungsschwelle der Öffentlichkeit. Sie markieren innerhalb des oben eingeführten Zyklus-Modells die Phase der Infragestellung etablierter Konfliktrahmen. Gleichwohl kann man mit einigem Recht davon ausgehen, dass der Großteil sozialer Akteure in der Eurozone solchen technischen Fragen eher indifferent gegenübersteht. Was ihnen aber weniger gleichgültig ist, sind die Anschlussfragen, die sich aus den gefundenen Antworten und politischen Reaktionen auf die genannten Entscheidungsprobleme ergeben. Das sind Fragen wie: Soll die Rettung maroder Banken zu Lasten ihrer Gläubiger oder zu Lasten der Steuerzahler gehen? In welchem Kompetenzverhältnis stehen europäische Finanzstabilisierungsinstrumente und nationale Budgethoheiten? Ist den Staatsschuldenproblemen einiger Euroländer wirksam mit Austeritätpolitik oder sinnvoller mit Investitionspolitik zu begegnen? Und wenn die Entscheidung zugunsten austeritätspolitischer Maßnahmen fällt: Was heißt das für wen konkret?

Jede einzelne dieser Entscheidungen ist nicht nur Ausdruck und Impuls institutionellen Wandels, ${ }^{51}$ sondern hat auch erhebliche Verteilungseffekte, die sich unter den Bedingungen knapper oder für knapp gehaltener Ressourcen in Verteilungskonflikten manifestieren. Der Zwang zum Schuldenabbau treibt in den stark krisenbetroffenen EU- beziehungsweise Euroländern nicht nur aggressive arbeitsmarkt- und sozialpolitische Abbauszenarien voran. Er führt auch zum rezessionsfördernden Wegbrechen der betroffenen Staaten als wirtschaftliche Akteure, und die dadurch massiv zunehmende Arbeitslosigkeit wird durch den (faktisch erzwungenen oder vorauseilend gehorsamen) Abbau öffentlicher Beschäftigung nochmals verschärft. Die Unsicherheiten auf den Finanzmärkten erschweren in der gesamten

50 Preunkert, Vobruba 2012.

51 Hofmann, Wessels 2013.

Leviathan, 42. Jg., 1/2014 
Eurozone die Kreditaufnahme der Unternehmen - mit negativen volkswirtschaftlichen Konsequenzen. Und in den krisengeschüttelten Staaten verschlechtern die Finanzmarktbedingungen und die rigiden Problemlösungsstrategien der Banken einhergehend mit zunehmender Arbeitslosigkeit und Einkommensverlusten - auch die Chancen privater Haushalte, eingegangene Kapitalverpflichtungen zu bedienen; Privatinsolvenzen oder auch Wohneigentumsverluste in großem Umfang sind die Folge. Die Niedrigstzins-Politik der EZB wiederum, mit der auf die wirtschaftlichen Schwierigkeiten in den krisenbetroffenen Euro-Volkswirtschaften reagiert wird, entfaltet ihre Wirkung unterschiedslos in der gesamten Eurozone mit der Folge, dass es (neben privaten) vor allem institutionellen Anlegern wie etwa Rentenfonds oder Lebensversicherungsgesellschaften nahezu unmöglich ist, das ihnen anvertraute Kapital rentabel, sicher und zugleich gesetzeskonform zu investieren.

Unabhängig von allen Unterschieden sind die sozialen Folgen der politischen Entscheidungen im Zuge der Staatsschuldenkrise also dramatisch. Darin stecken Gefahren und Chancen. Die Gefahren ergeben sich aus dem historisch immer wieder bestätigten Befund, dass in krisenhaften, bedrohlichen Situationen und in Phasen drastisch verschärfter wirtschaftlicher Unsicherheiten dem Bedürfnis nach Kontrollgewinn nicht zuletzt durch Intensivierung ethnozentrischer Einstellungen und Handlungsneigungen Rechnung getragen wird. ${ }^{52}$ Konflikttheoretisch ausgedrückt: In solchen Situationen steigt die Wahrscheinlichkeit wertebegründeter sozialer Abgrenzung und Dissoziation. Vor diesem Hintergrund sind Aufforderungen wie jene Giorgio Agambens zur (Re-)Separierung und Besinnung auf kulturelle Eigenheiten als wenigstens grob fahrlässig zu beurteilen. ${ }^{53}$ Denn sie erschweren die Einigung auf gemeinsame trans- und supranationale Konfliktrahmen. Stattdessen sollten Verteilungskonflikte als Verteilungskonflikte benannt und nicht $\mathrm{zu}-$ ungleich schwerer handhabbaren und tendenziell sozialdesintegrativen - Wertkonflikten stilisiert werden.

Das führt uns zu den Chancen, die sich aus den dramatischen sozialen Folgen des Krisenmanagements ergeben: Eine solche Dramatik begünstigt zugleich ihre Politisierung. Nationale Regulierung wird deutlich stärker als vor der Krise zum Gegenstand politischer Interessenbekundung sozialer Akteure; der Legitimationsbedarf des Krisenmanagements steigt stetig und in dem Maße, in dem seine Verteilungseffekte offensichtlich werden. Zugleich wird augenfällig, dass im Kontext der gegenwärtigen Krise und infolge der darauf reagierenden Austeritätspolitiken die Leistungs- und Kompensationsfähigkeiten der Umverteilungsarrangements in den am stärksten krisenbetroffenen Staaten drastisch abgenommen haben. Nationale Sicherungssysteme können nicht mehr als Interdependenzunterbrecher fungieren. Stattdessen suchen nun soziale Akteure verstärkt grenzüberschreitend und supranational nach Adressaten für ihre Sicherungserwartungen und Regulierungsbedürfnisse. Das heißt: Auch das Interesse an supranationaler Regulierung wird deutlich stärker und

52 Fritsche et al. 2011.

53 Agamben 2013. 
nachdrücklicher als vor der Krise. ${ }^{54}$ Dieser Nachdruck kann verschiedene Formen annehmen, aber er zeigt Erfolg: Erste Anzeichen einer institutionalisierten Sozialund Transferunion, die über Regionalförderungen und dergleichen hinausgeht, sind unübersehbar - genannt seien das Projekt einer gemeinsamen europäischen Arbeitslosenversicherung oder grenzüberschreitende Ausbildungssysteme zum Kampf gegen die Jugendarbeitslosigkeit in Südeuropa. Gerade sozialpolitische Maßnahmen als Konfliktrahmen, als eine spezifische Form des Umgangs mit - und der Regulierung von - Verteilungskonflikten können gesellschaftsbildende Effekte haben, sie können »die Triebkraft eines Vergesellschaftungsprozesses [sein], der sich als beständig fortschreitendes Wechselspiel der Institutionalisierung und Deinstitutionalisierung sozialer Beziehungen und Beziehungsmuster darstellt «. ${ }^{55}$

Indem die Krise der europäischen Integration zu einer Politisierung der politischen und ökonomischen europäischen Integration führt, erzwingt sie die Austragung transnationaler sozialer Konflikte und damit über kurz oder lang die Schaffung und Verfeinerung supranationaler Konfliktrahmen mit assoziativen, gesellschaftsbildenden Effekten. Sie macht Europa als Konfliktraum erfahrbar. Vor diesem Hintergrund spricht einiges dafür, dass die gegenwärtige Krise in Europa ein Vergesellschaftungsschub hin zu einer klarer erkennbaren europäischen Gesellschaft sein kann. In einer Entwicklung, die geprägt ist vom Wechselspiel zwischen Konflikten erster und zweiter Ordnung, kann sie als Trendbeschleuniger fungieren - und so die soziale Integration Europas vorantreiben.

\section{Literatur}

Agamben, Giorgio 2013. „Que l'Empire latin contre-attaque! «, in Libération vom 24. März 2013. www.liberation.fr/monde/2013/03/24/que-1-empire-latin-contre-attaque_890916 (Zugriff vom 31.10.2013).

Aubert, Vilhelm 1972. »Interessenkonflikt und Wertkonflikt: Zwei Typen des Konflikts und der Konfliktlösung " in Konflikt und Konfliktstrategie. Ansätze zu einer soziologischen Konflikttheorie, hrsg. v. Bühl, Walter L., S. 178-204. München: Nymphenburger Verlagshandlung.

Bach, Maurizio 2009. » Europäische Gesellschaft . Politische Integration und gesellschaftliche Desintegration in Europa ", in Mittelweg 36 18, 3, S. 17-29.

Blumenfeld, Kurt 1932. »Die zionist. Aufgabe im heutigen Deutschland «, in Jüdische Rundschau 37, 73/74, S. 353-354.

Coser, Lewis A. 1965. Theorie sozialer Konflikte. Neuwied: Luchterhand.

Dahrendorf, Ralf 1958. "Zu einer Theorie des sozialen Konflikts", in Hamburger Jabrbuch für Wirtschafts- und Gesellschaftspolitik 3, S. 76-92.

Dahrendorf, Ralf 1972. Konflikt und Freiheit. Auf dem Weg zur Dienstklassengesellschaft. München: Piper.

Dubiel, Helmut 1997. »Unversöhnlichkeit und Demokratie « in Was hält die Gesellschaft zusammen?, hrsg. v. Heitmeyer, Wilhelm, S. 425-444. Frankfurt a. M.: Suhrkamp.

Fehmel, Thilo 2013. Sicherungsbewahrung. Europas sozialpolitische Zukunft. Weinheim: Beltz Juventa.

54 Zürn 2013.

55 Lessenich 2008, S. 36.

Leviathan, 42. Jg., 1/2014 
Friedrichs, Jürgen 2007. »Gesellschaftliche Krisen. Eine soziologische Analyse « in Die Wahrnehmung von Krisenphänomenen. Fallbeispiele von der Antike bis in die Neuzeit, hrsg. v. Scholten, Helga, S. 13-26. Köln: Böhlau.

Fritsche, Immo; Jonas, Eva; Kessler, Thomas 2011. "Collective reactions to threat: implications for intergroup conflict and for solving societal crises ", in Social Issues and Policy Review 5, 1, S. 101-136.

Galtung, Johan 1970. »Theorien des Friedens « in Krieg oder Frieden. Wie lösen wir in Zukunft die politischen Konflikte?, hrsg. v. Schlemmer, Johannes, S. 133-148. München: Piper.

Hirschman, Albert O. 1994. »Wieviel Gemeinsinn braucht die liberale Gemeinschaft? «, in Leviathan 22, 2, S. 293-304.

Hofmann, Andreas; Wessels, Wolfgang 2013. »Tektonische Machtverschiebungen - die Krise als Auslöser und Verstärker des institutionellen Wandels", in Zeitschrift für Politik 60, 2, S. 220-241.

Honneth, Axel 2012. »Brutalization of the social conflict: struggles for recognition in the early 21st century ", in Distinktion: Scandinavian Journal of Social Theory 13, 1, S. 5-19.

Hooghe, Liesbet; Marks, Gary 2009. »A postfunctionalist theory of European integration: from permissive consensus to constraining dissensus «, in British Journal of Political Science 39, 1, S. 1-23.

Höreth, Marcus 2013. »Hemmungslos, aber ungefährlich? Der Gerichtshof der Europäischen Union als Verfassungsgericht im System der EU-Gewaltenteilung «, in Zeitschrift für Politik 60,1, S. 48-71.

Immerfall, Stefan 2013. »Über die Euro-Krise zur Fiskalunion? Mögliche Lehren für europäische Integration ", in Zeitschrift für Politik 60, 2, S. 195-206.

Jessop, Bob 2010. »The >return of the national state in the current crisis of the world market «, in Capital \& Class 34, 1, S. 38-43.

Knight, Jack 1997. Institutionen und gesellschaftlicher Konflikt. Tübingen: Mohr Siebeck.

Kühnhardt, Ludger. Hrsg. 2009. Crises in European integration. Challenge and response, 1945-2005. New York: Berghahn.

Lamping, Wolfram 2010. »Umkämpfte Grenzen. Über das Verhältnis von Sozial- und Wirtschaftspolitik auf EU-Ebene", in Sozialer Fortschritt 59, 5, S. 151-158.

Lessenich, Stephan 2008. Die Neuerfindung des Sozialen. Der Sozialstaat im flexiblen Kapitalismus. Bielefeld: transcript.

Lepsius, M. Rainer 1999. »Die Europäische Union. Ökonomisch-politische Integration und kulturelle Pluralität «, in Kultur, Identität, Europa. Über die Schwierigkeiten und Möglichkeiten einer Konstruktion, hrsg. v. Viehoff, Reinhold; Segers, Rien T., S. 201-222. Frankfurt a. M.: Suhrkamp.

Loo, Hans van der; Reijen, Willem van 1992. Modernisierung. Projekt und Paradox. München: dtv.

Mouffe, Chantal 2007. Über das Politische. Wider die kosmopolitische Illusion. Frankfurt a. M.: Suhrkamp.

Münch, Richard 2008 a. "Constructing a European society by jurisdiction «, in European Law Journal 14, 5, S. 519-541.

Münch, Richard 2008 b. Die Konstruktion der europäischen Gesellschaft. Zur Dialektik von transnationaler Integration und nationaler Desintegration. Frankfurt a. M.: Campus.

Offe, Claus 2005. »Soziale Sicherheit im supranationalen Kontext: Europäische Integration und die Zukunft des 'Europäischen Sozialmodells« in Welten des Kapitalismus. Institutionelle Alternativen in der globalisierten Ökonomie, hrsg. v. Miller, Max, S. 189-225. Frankfurt a. M.: Campus.

Preunkert, Jenny; Vobruba, Georg 2012. »Die Eurokrise - Konsequenzen der defizitären Institutionalisierung der gemeinsamen Währung « in Entfesselte Finanzmärkte. Soziologische Analysen des modernen Kapitalismus, hrsg. v. Kraemer, Klaus; Nessel, Sebastian, S. 201-224. Frankfurt a. M.: Campus.

Schroeder, Wolfgang; Weinert, Rainer 2003. »Europäische Tarifpolitik: Ein neues Politikfeld? «, in Gewerkschaften in Politik und Gesellschaft der Bundesrepublik Deutschland, hrsg. v. Schroeder, Wolfgang; Weßels, Bernhard, S. 565-587. Wiesbaden: Westdeutscher Verlag. 
Simmel, Georg 1992 [1894]. »Das Problem der Sociologie« in Aufsätze und Abhandlungen 1894 bis 1900. Georg Simmel Gesamtausgabe, Bd. 5, hrsg. v. Dahme, Heinz-Jürgen; Frisby, David P., S. 52-61. Frankfurt a. M.: Suhrkamp.

Simmel, Georg 1992 a [1908]. »Der Streit« in Soziologie. Untersuchungen über die Formen der Vergesellschaftung, hrsg. v. Rammstedt, Otthein, S. 284-382. Frankfurt a. M.: Suhrkamp.

Simmel, Georg 1992 b [1908]. »Die Erweiterung der Gruppe und die Ausbildung der Individualität" in Soziologie. Untersuchungen über die Formen der Vergesellschaftung, hrsg. v. Rammstedt, Otthein, S. 791-863. Frankfurt a. M.: Suhrkamp.

Steinert, Heinz 2007. »Sozialstaat und soziale Ausschließung « in Moderne (Staats)Bürgerschaft. Nationale Staatsbürgerschaft und die Debatten der Citizenship Studies, hrsg. v. Mackert, Jürgen, S. 147-165. Wiesbaden: VS Verlag für Sozialwissenschaften.

Taylor, Charles 2012. Ein säkulares Zeitalter. Berlin: Suhrkamp.

Vobruba, Georg 2012. Der postnationale Raum. Transformation von Souveränität und Grenzen in Europa. Weinheim: Beltz Juventa.

Vobruba, Georg 2013. Gesellschaftsbildung in der Eurokrise. Leipzig: Serie Europa - Europe Series 3/2013. www.uni-leipzig.de/leus/wp-content/uploads/2013-03-Vobruba-Gesellschaft sbildung-in-der-Eurokrise.pdf (Zugriff vom 31.10.2013)

Voigt, Stefan 2013. »How (not) to measure institutions «, in Journal of Institutional Economics 9 , 1, S. 1-26.

Walzer, Michael 1992. Sphären der Gerechtigkeit. Ein Plädoyer für Pluralität und Gleichheit. Frankfurt a. M.: Campus.

Whitehead, Alfred North 2000 [1927]. Kulturelle Symbolisierung. Frankfurt a. M.: Suhrkamp.

Wobbe, Theresa 2009. "Vom nation-building zum market-building. Der Wandel von Vergesellschaftungsformen im europäischen Integrationsprozess ", in Mittelweg 36 18, 3, S. 3-16.

Zürn, Michael 2013. »Nachwort: Die Finanz- und Schuldenkrise « in Die Politisierung der Weltpolitik. Umkämpfte internationale Institutionen, hrsg. v. Zürn, Michael; Ecker-Ehrhardt, Matthias, S. 413-425. Berlin: Suhrkamp. 
Zusammenfassung: Die derzeitige Verschärfung von innereuropäischen sozialen Konflikten wird überwiegend mit Sorge betrachtet. Der Aufsatz nimmt diese Sorge zum Anlass, den sozialen Konflikt im Sinne Georg Simmels als Grundlage von Vergesellschaftung und Gesellschaft zu rehabilitieren. Zunächst werden grundlegende Argumente für Bedingungen zusammentragen, unter denen Konflikte sozialintegrative Wirkungen haben können. Dann werden die aktuellen Entwicklungen in Europa zu den konflikttheoretischen Überlegungen in Beziehung gesetzt. Dabei geht es um die Frage, ob die Krise der europäischen Integration die soziale Integration Europas behindert oder begünstigt.

Stichworte: europäische Integration, sozialer Konflikt, Krise

\section{First and second order conflicts in Europe}

Summary: The currently intensified intra-European social conflicts are predominantly viewed with concern. This article intends to mitigate this concern by referring to Georg Simmel, who considered social conflicts as a specific base of socialization and society. First, fundamental arguments for conditions are gathered under which conflicts can have socially integrative effects. Then the current developments in Europe will be related to the conflict-theoretical considerations. This involves the question of whether the crisis of European integration is constricting or stimulating the social integration of Europe.

Keywords: European integration, social conflict, crisis

Autor

Dr. Thilo Fehmel

Lehrstuhlvertretung Soziale Institutionen und Sozialer Wandel

Universität Leipzig

Institut für Soziologie

Beethovenstraße 15

04107 Leipzig

fehmel@sozio.uni-leipzig.de 